\title{
AUTOMORPHISMS OF THE INTEGRAL GROUP RING OF $S_{n}$
}

\author{
GARY L. PETERSON
}

\begin{abstract}
In this paper it is shown that every normalized automorphism of the integral group ring of $S_{n}$ can be written as a group automorphism followed by conjugation by a unit in the group algebra of $S_{n}$ over the rationals.
\end{abstract}

1. Introduction. Throughout we will use $Z(G)$ to denote the integral group ring of a group $G$ and $\mathscr{\Re} Q(G)$ to denote the group of normalized automorphisms of $Z(G)$. That is, $\Re \mathscr{Q}(G)$ is the group of ring automorphisms of $Z(G)$ such that $f(g)$ has augmentation one for all $g$ in $G$. It is well known (see [2] or [3]) that it suffices to study normalized automorphisms over arbitrary automorphisms of $Z(G)$.

The major purpose of this paper is to consider $\Re Q(G)$ when $G=S_{n}$, the symmetric group on $n$ letters. In [2], Brown showed that $S_{n}$ is an $\mathcal{E}$. $\Re$. group for every $n=1,2, \ldots, 10$. That is, every normalized automorphism of $Z(G)$ can be written in the form $f=\tau_{u} \sigma$ where $\sigma$ is a group automorphism, $u$ is a unit in $Q(G)$ (the group algebra of $G$ over the rationals), and $\tau_{u}$ denotes conjugation by $u$ when $G=S_{n}, n=1, \ldots, 10$. In this paper, it will be shown that $S_{n}$ is an $\mathcal{E} . \Re$. group for any positive integer $n$.

It should be remarked that the study of $\mathcal{E} \cdot \mathcal{R}$. groups is not limited to $S_{n}$. The reader can find several types of metabelian $\mathcal{E}$. $R$. groups in [2], [4] and [6].

2. Action on class sums. Before looking at the case $G=S_{n}$, we will need some facts concerning the action of $\mathscr{Q} Q(G)$ on the class sums of a finite group $G$.

If $f$ is an element of $\mathscr{T} \mathscr{Q}(G)$ and $\bar{C}_{g}$ denotes the class sum of an element $g$ of $G$, then $f\left(\bar{C}_{g}\right)=\bar{C}_{x}$ for some $x$ in $G([2],[3],[6])$. Thus, $\mathcal{Q} Q(G)$ acts as a permutation group on the class sums of $G$. Further, let $\odot \odot(G)$ denote the kernel of this permutation representation. It follows that

$$
\mathcal{P P}(G)=\left\{\tau_{u} \mid u \text { is a unit in } Q(G) \text { normalizing } Z(G)\right\}
$$

by first extending every element of $\bigodot \mathcal{P}(G)$ to $Q(G)$, and then, since every element of $\bigodot \mathcal{P}(G)$ will fix the simple components of $Q(G)$, by applying the Noether-Skolem Theorem.

We will make use of the following two additional results.

Lemma 2.1. Let $f \in \mathfrak{R} Q(G)$ and $x, y, u, v$ be elements of $G$ such that

Received by the editors August 8, 1975.

AMS (MOS) subject classifications (1970). Primary 20C05, 16A26; Secondary 20B05, 20D45.

Key words and phrases. Integral group ring, symmetric group, normalized automorphism, group automorphism, augmentation, class sum. 
$f\left(\bar{C}_{x}\right)=\bar{C}_{u}$ and $f\left(\bar{C}_{y}\right)=\bar{C}_{v}$. Then, for some $g \in G$,

$$
f\left(\bar{C}_{x y}\right)=\bar{C}_{u v} \text {. }
$$

Proof. Note that $f\left(\bar{C}_{x y}\right)$ appears as a summand when $f\left(\bar{C}_{x} \bar{C}_{y}\right)$ is written as a linear combination of class sums. Also, $f\left(\bar{C}_{x} \bar{C}_{y}\right)$ is a linear combination of class sums comprised of elements of the form $u^{w} v^{z}$ where $w, z \in G$. Therefore, for some $w$ and $z$ in $G, f\left(\bar{C}_{x y}\right)=\bar{C}_{u_{v}^{w}}$ and we are done with $g=z w^{-1}$.

The second lemma is due to Brown [2].

Lemma 2.2. Let $f \in \Re Q(G)$ and suppose that $f\left(\bar{C}_{g}\right)=\bar{C}_{x}$ where $g$ and $x$ are elements of $G$. Then for every integer $n, f\left(\bar{C}_{g^{n}}\right)=\bar{C}_{x^{n}}$. Also, it follows that $|g|=|x|$.

3. Symmetric groups. We will now show that $S_{n}$ is an $\mathcal{E} \cdot \mathscr{R}$. group. In fact, we will actually see that $\Re \mathscr{Q}\left(S_{n}\right)=\mathcal{P} \mathcal{P}\left(S_{n}\right)$ for $n \neq 6$ which would have to hold since every group automorphism of $S_{n}$ is inner when $n \neq 6$ ([5], Theorem 11.4.8).

We first record two results about $S_{n}$. The first result is Exercise 11.4.11(a) of [5]. The second is a well-known result about the order of conjugacy classes of $S_{n}$ and can be found, for instance, in [1].

Lemma 3.1. Let $n>2, n \neq 6$. Then an element $x$ of $S_{n}$ is a 2-cycle if and only if $|x|=2$ and $\max \left|x x^{y}\right|=3$ where $y$ ranges over the elements of $S_{n}$.

LEMMA 3.2. Let $g \in S_{n}$ and suppose that $g$ is a product of disjoint $k_{1} 1$-cycles, $k_{2} 2$-cycles, $\ldots, k_{n} n$-cycles. Then the order of $C_{g}, C_{g}$ the conjugacy class of $g$, is given by

$$
\left|C_{g}\right|=n ! /\left(k_{1} ! 1^{k_{1}} k_{2} ! 2^{k_{2}} \ldots k_{n} ! n^{k_{n}}\right)
$$

We next show

LEMMA 3.3. Let $f$ be a normalized automorphism of $Z\left(S_{n}\right)$ and suppose that $n>2, n \neq 6$. Let $g$ in $S_{n}$ be a product of disjoint transpositions. Then $f\left(\bar{C}_{g}\right)=\bar{C}_{g}$.

Proof. Let $t$ denote the number of transpositions appearing in $G$. We may assume that $g$ has the form $g=(1,2)(3,4) \ldots(2 t-1,2 t)$. Let $f\left(\bar{C}_{g}\right)=\bar{C}_{x}$, $x \in G$. To show $\bar{C}_{x}=\bar{C}_{g}$ we proceed by induction on $t$.

If $t=1$, let $u \in S_{n}$. By Lemma 2.1, we can find $v$ in $S_{n}$ such that $f\left(\bar{C}_{g g^{u}}\right)=\bar{C}_{x x^{v}}$. Conversely, given $v$ in $S_{n}$ we can also find $u$ in $S_{n}$ satisfying the above equation. Thus by Lemma $2.2, \max \left|x x^{v}\right|=3$ as $v$ runs over $S_{n}$. Thus $x$ is a transposition by Lemma 3.1 since $|x|=2$ by Lemma 2.2.

In the general case we have

$$
f\left(\bar{C}_{(1,2)(3,4) \ldots(2 t-3,2 t-2)}\right)=\bar{C}_{(1,2)(3,4) \ldots(2 t-3,2 t-2)}
$$

and $f\left(\bar{C}_{(2 t-1,2 t)}\right)=\bar{C}_{(2 t-1,2 t)}$. Hence by Lemma 2.1 ,

$$
f\left(\bar{C}_{g}\right)=\bar{C}_{(1,2)(3,4) \ldots(2 t-3,2 t-2)(2 t-1,2 t)^{y}}
$$

for some $y \in S_{n}$.

If we can show that $(2 t-1,2 t)^{y}$ is disjoint from $(1,2)(3,4) \ldots(2 t-3$, $2 t-2)$, we will be done, so suppose this is not the case. If $(2 t-1,2 t)^{y}$ has 
one letter in common with $(1,2) \ldots(2 t-3,2 t-2)$, it follows that $(1$, $2) \ldots(2 t-3,2 t-2)(2 t-1,2 t)^{y}$ is a product of disjoint cycles which are transpositions and a 3 -cycle. But then 3 divides $|x|$ which is impossible.

Next, suppose $(2 t-1,2 t)^{y}$ has two letters in common with one transposition of $(1,2) \ldots(2 t-3,2 t-2)$. Then $x$ is a product of disjoint transpositions and has fewer transpositions than $g$ does. Thus $f$ fixes $\bar{C}_{x}$ and so $f\left(\bar{C}_{g}\right) \neq \bar{C}_{x}$.

The final possibility would be for $(2 t-1,2 t)^{y}$ to have one letter in common with two different transpositions of $(1,2) \ldots(2 t-3,2 t-2)$. But then $(1,2) \ldots(2 t-3,2 t-2)(2 t-1,2 t)^{y}$ is a product of disjoint cycles which are transpositions and a 4 -cycle. Hence 4 divides $|x|$, again a contradiction.

We now come to

THEOREM 3.4. $S_{n}$ is an $\mathcal{E}$. R. group for every positive integer $n$. Moreover, $\Re \mathscr{Q}\left(S_{n}\right)=\mathcal{P}\left(S_{n}\right)$ for every $n \neq 6$.

Proof. By the results of [2], we may assume $n>2$ and $n \neq 6$. Let $f \in \mathscr{T} Q(G)$ and note that it suffices to show that $f \in \mathcal{P}(G)$. To accomplish this, we set

$$
N=\left\{g \in S_{n} \mid f\left(\bar{C}_{g}\right) \neq \bar{C}_{g}\right\}
$$

and show that $N$ is the empty set.

Suppose $N$ is nonempty. We pick a "minimal element" $g$ of $N$ satisfying the following properties:

(1) Suppose $g$ has its largest cycle of smallest length among the elements of $N$. Let $h$ denote the length of its largest cycle.

(2) Suppose that $g$ has the fewest number of cycles of length $h$ among the elements of $N$ satisfying (1).

Note that we have $h \geqslant 3$ by Lemma 3.3.

Write $g=\beta_{1} \beta_{2} \ldots \beta_{r}$ where the $\beta_{i}$ are disjoint cycles and $2 \leqslant\left|\beta_{i}\right|$ $\leqslant\left|\beta_{i+1}\right|$. Also, assume that $\beta_{r}=(1,2, \ldots, h)$. Let $\gamma_{r}=(1,2, \ldots, h-1)$, then $g=\beta_{1} \ldots \beta_{r-1} \gamma_{r}(1, h)$. Also, by the minimality of $g$,

$$
f\left(\bar{C}_{\beta_{1} \ldots \beta_{r-1} \gamma_{r}}\right)=\bar{C}_{\beta_{1} \ldots \beta_{r-1} \gamma_{r}}
$$

and by Lemma 3.3, $f\left(\bar{C}_{(1, h)}\right)=\bar{C}_{(1, h)}$. Thus by Lemma 2.1

$$
f\left(\bar{C}_{g}\right)=\bar{C}_{\beta_{1} \ldots \beta_{r-1} \gamma_{r} \alpha}
$$

where $\alpha=(1, h)^{x}$ for some $x \in S_{n}$. Let $y=\beta_{1} \ldots \beta_{r-1} \gamma_{r} \alpha$. We will obtain a contradiction by showing $\bar{C}_{g}=\bar{C}_{y}$. The proof of this is broken into the following cases:

Case 1. If $(1, h)^{x}$ is disjoint from $\beta_{1} \ldots \beta_{r-1} \gamma_{r}$.

In this case, $f$ must fix $\bar{C}_{y}$ by the minimality of $g$ since $y$ has fewer cycles of length $h$ than $g$ with its largest cycle of length less than or equal to $h$. But then $f\left(\bar{C}_{g}\right) \neq f\left(\bar{C}_{y}\right)=\bar{C}_{y}$ and so this case cannot occur.

Case 2. If $(1, h)^{x}$ has one letter in common with $\beta_{1} \ldots \beta_{r-1} \gamma_{r}$.

Suppose $\left(n_{1}, n_{2}, \ldots, n_{t}\right)$ is the cycle of $\beta_{1} \ldots \beta_{r-1} \gamma_{r}$ where the common letter occurs. Then $(1, h)^{x}=\left(n_{j}, a\right)$ where $a$ does not appear in $\beta_{1} \ldots \beta_{r-1} \gamma_{r}$. 
Also, note that

$$
\left(n_{1}, \ldots, n_{t}\right)\left(n_{j}, a\right)=\left(n_{1}, \ldots, n_{j-1}, a, n_{j}, \ldots, n_{t}\right) .
$$

If $t<h-1, y$ would have fewer cycles of length $h$ with its largest cycle of length less than or equal to $h$. Thus by the minimality of $g, \bar{C}_{y}$ is fixed by $f$ and so $f\left(\bar{C}_{g}\right) \neq \bar{C}_{y}$.

If $t>h-1$, then $t=h$. But then, $y$ has one more cycle of length $h-1$ than $g$ does. Thus $y^{h}$ has one more cycle of length $h-1$ than $g^{h}$ does. Therefore, by the minimality of $g$,

$$
f\left(\bar{C}_{g^{h}}\right)=\bar{C}_{g^{h}}=\bar{C}_{y^{h}}
$$

which is impossible.

Thus we have $t=h-1$. But then $y$ has the same cycle structure as $g$, and hence $\bar{C}_{g}=\bar{C}_{y}$.

Case 3 . If $(1, h)^{x}$ has two letters in common with $\beta_{1} \ldots \beta_{r-1} \gamma_{r}$.

First, suppose that $(1, h)^{x}$ has two letters in common with one cycle of $\beta_{1} \ldots \beta_{r-1} \gamma_{r}$. Let $\left(n_{1}, \ldots, n_{t}\right)$ denote this cycle and suppose $(1, h)^{x}=\left(n_{j}, n_{s}\right)$ where $j<s$. Then

$$
\begin{aligned}
\left(n_{1}, \ldots, n_{t}\right) & \left(n_{j}, n_{s}\right) \\
& =\left(n_{1}, \ldots, n_{j-1}, n_{s}, n_{s+1}, \ldots, n_{t}\right)\left(n_{j}, n_{j+1}, \ldots, n_{s-1}\right)
\end{aligned}
$$

where the first cycle on the right side of the above equation is taken to be the identity when $j=1$ and $s=t$. Hence, $y$ has fewer cycles of length $h$ with its largest cycle of length less than or equal to $h$. By the minimality of $g, f$ fixes $\bar{C}_{y}$ so that $f\left(\bar{C}_{g}\right) \neq \bar{C}_{y}$.

Thus, $(1, h)^{x}$ must have its letters in common with two cycles of $\beta_{1} \ldots \beta_{r-1} \gamma_{r}$. Let $\left(n_{1}, \ldots, n_{t}\right)$ and $\left(n_{1}^{\prime}, \ldots, n_{s}^{\prime}\right)$ denote these cycles where $t \leqslant s$. Then $(1, h)^{x}$ has the form $\left(n_{i}, n_{j}^{\prime}\right)$ and

$$
\begin{aligned}
\left(n_{1}, \ldots, n_{t}\right)\left(n_{1}^{\prime}, \ldots, n_{s}^{\prime}\right)\left(n_{i}, n_{j}^{\prime}\right) & \\
& =\left(n_{1}, \ldots, n_{i-1}, n_{j}^{\prime}, n_{j+1}^{\prime}, \ldots, n_{s}^{\prime}, n_{1}^{\prime}, \ldots, n_{j}^{\prime}, n_{i}, \ldots, n_{t}\right) .
\end{aligned}
$$

If neither $s$ nor $t$ is $h-1$, then $y$ has one more cycle of length $h-1$ than $g$ does. Thus, $y^{h}$ has one more cycle of length $h-1$ than $g^{h}$ does. But then, by the minimality of $g, f\left(\bar{C}_{g^{h}}\right)=\bar{C}_{g^{h}} \neq \bar{C}_{y^{h}}$. Hence, either $s$ or $t$ is $h-1$ and $s+t>h$. 3.2 ,

Suppose that $g$ has $k_{1} 1$-cycles, $k_{2}$ 2-cycles, . , $k_{h} h$-cycles. By Lemma

$$
\left|C_{g}\right|=n ! / k_{1} ! k_{2} ! 2^{k_{2}} \ldots k_{h} ! h^{k_{h}} .
$$

If $s=h-1$ and $t<s$, then

$$
\begin{aligned}
\left|C_{y}\right|=n ! /( & k_{1} ! k_{2} ! 2^{k_{2}} \ldots k_{t-1} !(t-1)^{k_{t-1}}\left(k_{t}-1\right) ! t^{k_{t}-1} \\
& \left.\quad k_{t+1} !(t+1)^{k_{t+1}} \ldots k_{h-1} !(h-1)^{k_{h-1}}\left(k_{h}-1\right) ! h^{k_{h}-1}(s+t)\right) .
\end{aligned}
$$

Since $\left|C_{g}\right|=\left|C_{y}\right|, s+t=k_{t} t k_{h} h$. But this is impossible since $t h>s+t$.

If $t=h-1$ and $s=h-1$,

$$
\left|C_{y}\right|=n ! /\left(k_{1} ! k_{2} ! 2^{k_{2}} \ldots\left(k_{h-1}-1\right) !(h-1)^{k_{h-1}-1}\left(k_{h}-1\right) ! h^{k_{h}-1}(s+t)\right) \text {. }
$$


Thus $\left|C_{g}\right|=\left|C_{y}\right|$ implies $s+t=k_{h-1}(h-1) k_{h} h$. But this is also impossible since $(h-1) h>s+t$.

Finally, if $t=h-1$ and $s=h$,

$$
\left|C_{y}\right|=n ! /\left(k_{1} ! k_{2} ! 2^{k_{2}} \ldots k_{h-1} !(h-1)^{k_{h-1}}\left(k_{h}-2\right) ! h^{k_{h}-2}(s+t)\right) .
$$

Again, since $\left|C_{g}\right|=\left|C_{y}\right|, s+t=\left(k_{h}-1\right) k_{h} h^{2}$. Once more, this is impossible since $h^{2}>s+t$ and hence Case 3 cannot occur.

Thus, since only Case 2 occurs and since $\bar{C}_{g}=\bar{C}_{y}$ in this case, $N$ is the empty set and $f \in \mathcal{P}(G)$.

ACKNowledgement. This paper is taken from Chapter $V$ of the author's doctoral thesis written under the supervision of Professor J. E. Adney whom I wish to thank for his encouragement and guidance.

\section{REFERENCES}

1. H. Boerner, Representations of groups, 2nd English ed., North-Holland, Amsterdam; American Elsevier, New York, 1970. MR 42 \#792.

2. C. F. Brown, Automorphisms of integral group rings, Ph. D. Thesis, Michigan State University, 1971.

3. G. L. Peterson, Automorphisms of integral group rings. I Arch. Math. (to appear).

4. , Automorphisms of integral group rings. II (to appear).

5. W. R. Scott, Group theory, Prentice-Hall, Englewood Cliffs, N. J., 1964. MR 29 \#485.

6. S. K. Sehgal, On the isomorphism of integral group rings. I, Canad. J. Math. 21 (1969), 410-413. MR 41 \#366.

Department of Mathematics, Michigan State University, East Lansing, Michigan 48824

Department of Mathematics, Southern Illinois University, Carbondale, Illinois 62901 (Current address) 